\title{
Comparative study of carcinoembryonic antigen in rheumatoid synovium, tumour, and normal adult lun
}

\author{
ARLEEN UNGER, G. S. PANAYI, AND N. H. TIDMAN \\ From the Department of Medicine, Guy's Hospital Medical School, London SE1
}

SUMMARY Material reacting like carcinoembryonic antigen (CEA) in the radioimmunoassay h\% been extracted from rheumatoid synovial membranes. This CEA activity has been compared to that found in hepatic metastases from colorectal tumours and in normal adult lung. The antigen in the rheumatoid synovium has been shown to be more sensitive to perchloric acid and to isolate with $\$$ lower weight than that derived from the tumour and lung. Immunodiffusion studies with anti-CEA indicate that the CEA-like determinants in the rheumatoid synovium have partial identity with tumour CEA and that a significant proportion of them are associated with large molecular weight material. Production of an antiserum to these CEA-like components in the rheumatoid synoviura should enable further identification of their relationship to tumour CEA and might allow a bettep judgement of whether or not they represent the expression of neoantigens in the disease.

Plasma levels of carcinoembryonic antigen (CEA) have been shown to be significantly higher in patients with seropositive rheumatoid arthritis (RA) than in normal subjects (Unger et al., 1974). In addition, material reacting like CEA in the radioimmunoassay was found in extracts of rheumatoid synovial membranes but not in normal synovium (Unger et al., 1975). This finding might be taken as evidence of expression of a neoantigen in the inflamed synovium which could be of some significance in the aetiopathology of the disease. However, many studies have shown that CEA in malignant and normal adult tissues can be closely associated with other antigens, such as a nonspecific cross-reacting antigen known either as NCA (Kleist et al., 1972) or CEX (Darcy et al., 1973), and blood group substances (Simmons and Perlmann, 1973; Feizi et al., 1975) which may interfere in the CEA radioimmunoassay. It is essential therefore to characterize the CEA activity in rheumatoid synovial membranes and determine its relationship, if any, to some of these known cross-reacting substances.

CEA activity in rheumatoid synovial membranes has been compared in terms of extractability in perchloric acid and molecular weight (as assessed by gel filtration) to that derived from human tumours and normal adult tissue.

Accepted for publication June 1, 1976

Correspondence to Dr. A. Unger

\section{Materials and methods}

EXTRACTION OF TISSUES

Rheumatoid synovial membranes (RA/SM) wer" obtained fresh as synovectomy specimens. Hepatie metastases from colorectal tumours and norma adult lung ( $\mathrm{N}$ lung) were obtained at autopsy. AP्P specimens were stored at $-20^{\circ} \mathrm{C}$ until required. A tissues were extracted in an identical manner. Tissue was first homogenized in distilled wate? (3 volumes/g wet weight of tissue) and the homo genate centrifuged at $1000 \mathrm{~g}, 4^{\circ} \mathrm{C}$ for 20 minutes to remove insoluble material. Half the total volume of the supernatant was freeze-dried and labelled 'wate extract' $\left(\mathrm{H}_{2} \mathrm{O}\right.$ extract). To the remaining supernatant an equal volume of $1.2 \mathrm{~N}$ perchloric acid was addec, stirred at room temperature for 30 minutes, the clarified by centrifugation at $2000 \mathrm{~g}, 4^{\circ} \mathrm{C}$ for 20 minutes. The supernatant was neutralized by tho dropwise addition of $10 \mathrm{~N}$ sodium hydroxide, diar lysed against running tap water for 2 days, then overo night against distilled water. The extract was freeze dried and labelled 'perchloric acid extract' (PCA extract).

GEL FILTRATION CHROMATOGRAPHY Sepharose 4B (Pharmacia Ltd.) was packed in column $2.6 \times 100 \mathrm{~cm}$ and equilibrated with phost phate buffer $(0.01 \mathrm{~mol} / \mathrm{l}$ phosphate, $0.15 \mathrm{~mol}$ 居 sodium chloride, $0.01 \mathrm{~mol} / \mathrm{l}$ edetic acid (EDTA) 
and $0.05 \%$ sodium azide adjusted to $\mathrm{pH} 7.0$ with $1 \mathrm{~N} \mathrm{NaOH}$ ). The column was then calibrated with Dextran blue, ${ }^{125}$ I-CEA, and bovine serum albumin, their peaks of elution denoted by arrows in Figs. 1 and 2. Approximately $1 \mathrm{~g}$ of each of the freezedried $\mathrm{H}_{2} \mathrm{O}$ extracts and $0 \cdot 1 \mathrm{~g}$ of the PCA extracts were separately resuspended in $5 \mathrm{ml}$ phosphate buffer, mixed by continuous rolling for 2 hours at room temperature, and centrifuged at $20000 \mathrm{~g}, 4^{\circ} \mathrm{C}$ for 30 minutes to remove insoluble material. $4 \mathrm{ml}$ of each extract was separately chromatographed on the same column of Sepharose 4B. The column was pumped at $15 \mathrm{ml} / \mathrm{h}$ and 15 -minute fractions collected. In the case of the $\mathrm{H}_{2} \mathrm{O}$ and PCA extracts of the tumour, individual fractions were assayed for CEA to determine the peak of CEA activity (Figs. 1 and 2). Fractions which included the greater part of this peak were pooled and concentrated by ultrafiltration. The remaining fractions before and after the CEA peak were also pooled and concentrated so that 2 fractions (A and B) of higher, and 1 fraction (D) of lower, molecular weight than CEA (fraction C) were obtained (Figs. 1 and 2). Since the RA/SM and $\mathrm{N}$ lung extracts contained relatively low amounts

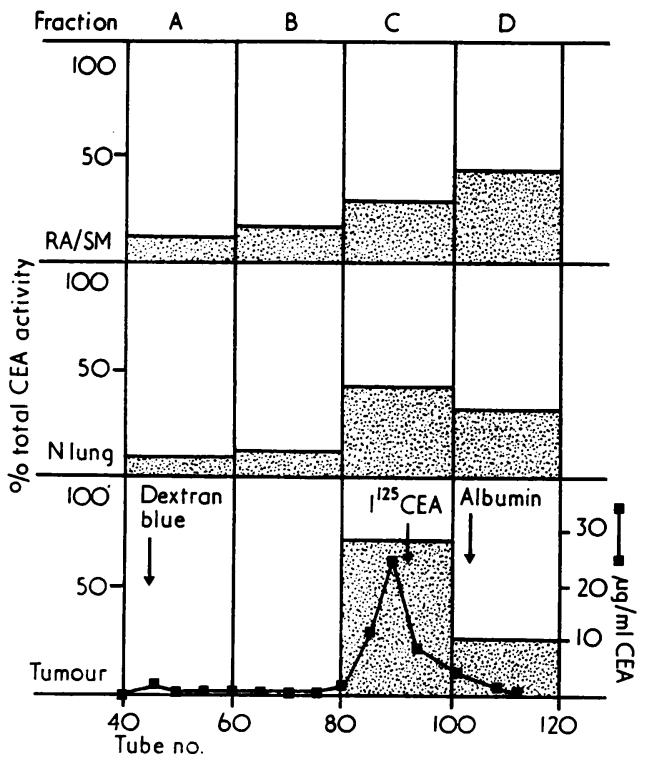

Fig. 1 Sepharose $4 B$ chromatography of water extracts. Shaded areas represent percentage of total $C E A$ activity in each of the four fractions $(A, B, C, D)$; $\square=C E A \mu \mathrm{g} / \mathrm{ml}$ in individual fractions from tumour extract; $R A / S M=$ rheumatoid synovial membranes; $N$ lung = normal lung.

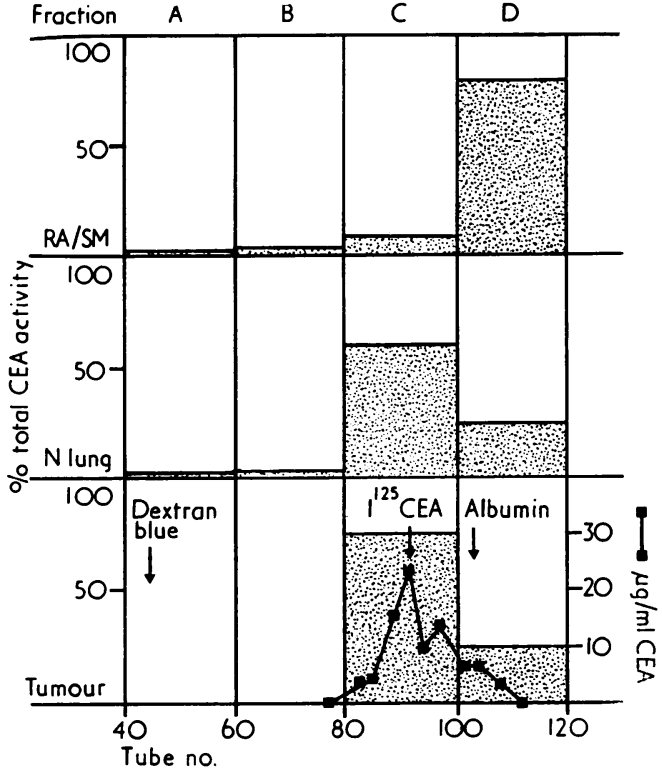

Fig. 2 Sepharose $4 B$ chromatography of perchloric acid extracts. Shaded areas represent percentage of total $C E A$ activity in each of the four fractions $(A, B, C, D)$; $\square=C E A \mu \mathrm{g} / \mathrm{ml}$ in individual fractions from tumour extract.

of CEA activity, the individual fractions were not assayed for CEA but instead were pooled and concentrated as for the tumour fractions A, B, C, and $D$. The CEA estimated by radioimmunoassay in each of the fractions (A, B, C, and D) was expressed as a percentage of the total CEA activity eluting from the gel filtration of each extract.

CEA RADIOIMMUNOASSAY

This was performed by the double antibody technique of Egan et al. (1972), as modified by Laurence et al. (1972).

IMMUNODIFFUSION IN AGAR

Immunodiffusion reactions were performed in $1 \%$ Ionagar No. $2(\mathrm{w} / \mathrm{v})$ in phosphate buffer $\mathrm{pH} 6.8$ on glass plates $(8 \mathrm{~cm} \times 8 \mathrm{~cm})$. The plates were maintained in a moist chamber at room temperature for 3 days, then photographed.

\section{Results}

EXTRACTION BY PERCHLORIC ACID

The CEA activity of the $\mathrm{H}_{2} \mathrm{O}$ and PCA extracts was estimated by radioimmunoassay and the total CEA activity calculated and expressed as $\mu \mathrm{g} \mathrm{CEA} / \mathrm{g}$ 
freeze-dried tissue. This enabled the percentage CEA activity extracted by perchloric acid from each tissue to be calculated (Table 1). Extraction by perchloric acid resulted in a loss of CEA activity from the three tissues, the greatest loss $(85 \%)$ being from the RA/SM, while those from the tumour and $\mathrm{N}$ lung were very similar $(50 \%$ and $53 \%$, respectively).

Table 1 CEA activity in water and perchloric acid extracts

\begin{tabular}{lccc}
\hline & $\begin{array}{l}\text { Water } \\
(\mu g C E A / g \text { FD } \\
\text { tissue })\end{array}$ & $\begin{array}{l}\text { Perchloric acid } \\
(\mu g C E A / g F D \\
\text { tissue })\end{array}$ & $\begin{array}{l}\% \text { extracted by } \\
\text { acid }\end{array}$ \\
\hline RA/SM & 1.55 & 0.24 & 15.5 \\
N lung & 11.70 & 5.50 & 47.0 \\
Tumour & 2500.0 & 1264.0 & 50.6 \\
\hline
\end{tabular}

$F D=$ freeze dried.

SEPHAROSE 4B CHROMATOGRAPHY

The percentages of total CEA activity found in fractions A, B, C, and D of the chromatographed $\mathrm{H}_{2} \mathrm{O}$ and PCA extracts are shown in Figs. 1 and 2, respectively. Calibration of the Sepharose 4B column with radio-iodinated CEA and estimation of individual fractions from the tumour extracts indicated that the major portion of CEA should be found in fraction $\mathrm{C}$. In the $\mathrm{H}_{2} \mathrm{O}$ extract of RA/SM, however, only $28 \%$ of the total CEA activity located in this fraction, the highest proportion ( $42 \%$ ) being found in fraction $D$ and a considerable amount $(30 \%)$ in the higher molecular weight fractions $\mathrm{A}$ and B. A similar significant proportion (22\%) was also found in fractions $A$ and $B$ of the $N$ lung $\mathrm{H}_{2} \mathrm{O}$ extract, the remainder being almost evenly divided between fractions $\mathrm{C}$ and $\mathrm{D}(43 \%$ and $34 \%$, respectively).

After perchloric acid extraction, the CEA activity in fractions $\mathbf{A}$ and $\mathbf{B}$ of $\mathbf{N}$ lung became almost negligible and the resulting profile of elution was almost indistinguishable from that of the tumour extract, $70 \%$ and $74 \%$ respectively being found in fractions C. Acid extraction of RA/SM also resulted in a loss of CEA activity from fractions $A$ and $B, \stackrel{\oplus}{?}$ but unlike $\mathrm{N}$ lung and tumour the major proportion $\overrightarrow{\bar{F}}$ (82\%) located in fraction $\mathrm{D}$, only $9 \%$ being found in fraction C.

\section{IMMUNODIFFUSION STUDIES}

Preliminary experiments showed that faint but $\stackrel{\mathbb{\alpha}}{2}$ definite precipitin lines were obtained with some of the RA/SM fractions when $70 \mu l$ of anti-CEA was $\overrightarrow{0}$ reacted with $15 \mu$ l of the fractions. These conditions $\overrightarrow{-}$ were used throughout these studies and were alsow applied to the tumour and $\mathbf{N}$ lung fractions. Table@ 2 shows the number of precipitins obtained and also? the concentration of CEA (estimated by the radio- $\omega$ immunoassay) in each fraction.

Some of the immunodiffusion results are shown ing Fig. 3A (diagrammatically represented in Fig. 3B). 응 Faint but definite double precipitins, having com-plete identity with one another, were observed T between anti-CEA (well 12) and fractions $B$ of the $\mathrm{H}_{2} \mathrm{O}$ and PCA extracts of RA/SM (wells 1 and 2). The inner line (nearer the antiserum well 12) crossed completely the heavier inner precipitin $\overrightarrow{0}$ produced with purified tumour CEA in the adjacent $\forall$ well (well 3) but its exact relationship to the sharp innermost precipitin of the CEA reaction could not be determined. The outer line (nearer the antigen well 2) of the RA/SM reaction appeared to touch the CEA precipitin giving a reaction of partial $\frac{}{\mathbb{Q}}$ identity. With anti-CEX (well 10), the only fraction $\varrho$ of the RA/SM extracts to react was fraction D of the water extract which gave a sharp single precipitin 3 line (wells 5 and 7). These precipitin lines were much nearer the antiserum well (well 10) than the CEX reaction in the adjacent well (well 4). The reactions obtained with anti-CEA (wells 11 and 12) anti-CEX (well 10), CEA (well 3), and a mixture of CEA and 3 CEX (well 4) indicate that the anti-CEA has anc anti-CEX component, and conversely that the antiCEX carries an anti-CEA contaminant (wells 8 , 0 $10,11)$.

Table 2 Immunodiffusion reactions with anti-CEA and fractions from Sepharose $4 B$

\begin{tabular}{|c|c|c|c|c|c|c|c|c|}
\hline & \multicolumn{2}{|l|}{ Fraction $A$} & \multicolumn{2}{|l|}{ Fraction $B$} & \multicolumn{2}{|l|}{ Fraction $C$} & \multicolumn{2}{|l|}{ Fraction $D$} \\
\hline & $C E A \mu g / m l$ & $I D$ & $C E A \mu g / m l$ & $I D$ & $C E A \mu g / m l$ & $I D$ & $C E A \mu g / m l$ & $I D$ \\
\hline $\begin{array}{l}\text { Water extracts } \\
\text { RA/SM } \\
\text { Nlung } \\
\text { Tumour }\end{array}$ & $\begin{array}{l}0 \cdot 2 \\
0 \cdot 3 \\
4 \cdot 9\end{array}$ & $\begin{array}{l}- \\
+ \\
+\end{array}$ & $\begin{array}{l}0 \cdot 4 \\
0 \cdot 3 \\
2 \cdot 2\end{array}$ & $\begin{array}{l}++ \\
+ \\
+\end{array}$ & $\begin{array}{r}0.4 \\
1.6 \\
410\end{array}$ & $\begin{array}{l}- \\
++\end{array}$ & $\begin{array}{r}0.3 \\
0.4 \\
136.0\end{array}$ & $\begin{array}{l}- \\
\bar{t}+\end{array}$ \\
\hline $\begin{array}{l}\text { Perchloric acid extracts } \\
\text { RA/SM } \\
\text { Nlung } \\
\text { Tumour }\end{array}$ & $\begin{array}{l}0.01 \\
0.04 \\
3.02\end{array}$ & $\begin{array}{l}- \\
-\end{array}$ & $\begin{array}{l}0.04 \\
0.05 \\
7 \cdot 2\end{array}$ & $\begin{array}{l}++ \\
- \\
-\end{array}$ & $\begin{array}{c}0.04 \\
0 \cdot 4 \\
1000 \cdot 0\end{array}$ & $\begin{array}{l}t+ \\
- \\
t+t\end{array}$ & $\begin{array}{l}0 \cdot 21 \\
0 \cdot 13 \\
383 \cdot 2\end{array}$ & $\begin{array}{l}- \\
+ \\
+\end{array}$ \\
\hline
\end{tabular}

ID = immunodiffusion; $-=$ no precipitin line; $+=1$ precipitin line; ++2 precipitin lines; +++3 precipitin lines 

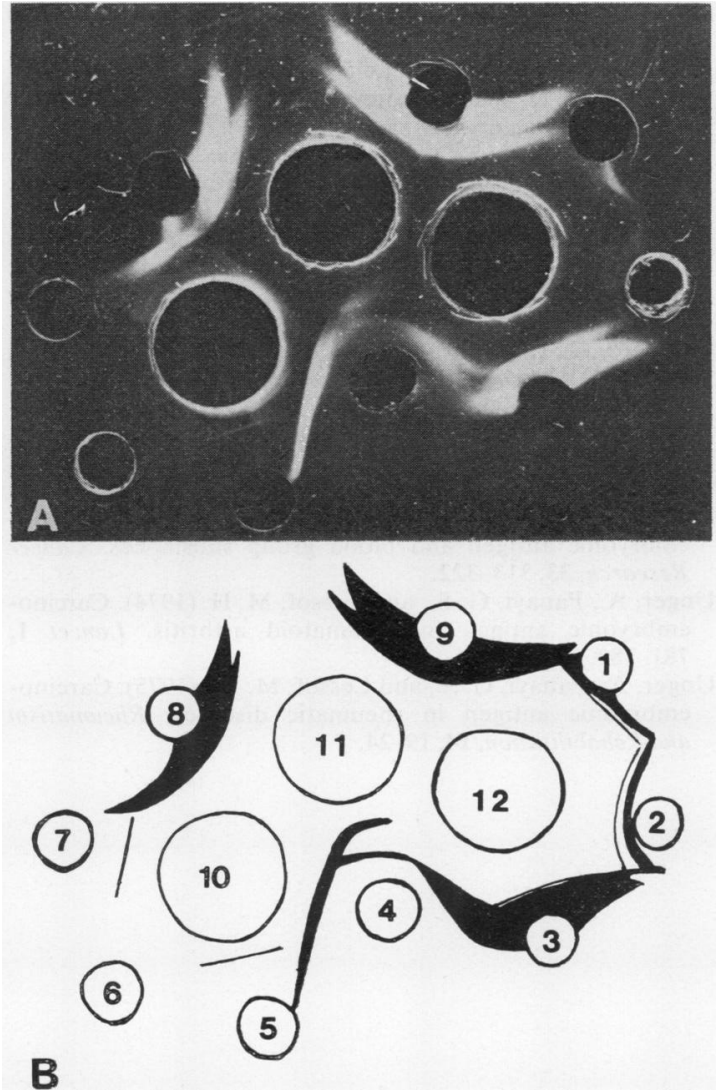

Fig. 3 (A) Double immunodiffusion of RA/SM, and tumour fractions with anti-CEA and anti-CEX. (B) Diagrammatic representation of $(A)$. Wells (I) PCA extract $\mathrm{RA} / \mathrm{SM}$ fraction $\mathrm{B} ;(2,6) \mathrm{H}_{2} \mathrm{O}$ extract $\mathrm{RA} / \mathrm{SM}$ fraction $B ;(3,8,9)$ tumour $C E A$; (4) tumour $C E A$ and $C E X ;(5,7) \mathrm{H}_{2} \mathrm{O}$ extract $\mathrm{RA} / \mathrm{SM}$ fraction $\mathrm{D} ;(10)$ anti-CEX; $(11,12)$ anti-CEA.

\section{Discussion}

This study has shown that CEA activity in RA/SM differs from that found in a tumour of colorectal origin and normal adult lung in several respects. It was destroyed by perchloric acid extraction to a much greater extent than that from either tumour or $\mathrm{N}$ lung. Sensitivity to perchloric acid has been noted to be a feature of the cross-reacting antigen, CEX (Kleist et al., 1972), and neutralization of the acid before dialysis was advocated to prevent its destruction. However, the heavy loss of CEA activity from RA/SM was seen despite this neutralization step being included in the extraction procedure.

Sepharose 4B chromatography of the $\mathrm{H}_{2} \mathrm{O}$ extracts showed a certain degree of similarity between
RA/SM and $\mathrm{N}$ lung in that relatively high proportions, $30 \%$ and $22 \%$ respectively, of their total CEA activities eluted in the fractions (A and B) of higher molecular weight than CEA. However, after perchloric acid extraction of these two tissues, the proportion of total CEA activity found in these fractions (A and $B$ ) and in fraction $C$ of the RA/SM was very much reduced. This might indicate that the large molecular weight material was split into smaller units during acid treatment or that it was lost, possibly by precipitation. Evidence that perchloric acid extraction of CEA from tumour material occasionally resulted in complete precipitation of the antigen was presented by Dyce and Haverback (1974). Excessive precipitation would certainly account for the poor recovery of CEA activity in the PCA extract of RA/SM. Alternatively. the localization of the major portion of the CEA activity in the lower molecular weight region (fraction D) might indicate that at least part of the antigen was split into smaller fragments. No evidence was obtained from immunodiffusion studies of CEA being present in this fraction, and although there was a reaction with anti-CEX it appeared to have no identity with CEX in the adjacent well. However, CEX with a molecular weight of 60000 would be expected to isolate in this fraction.

The immunodiffusion studies also showed that the high molecular weight fractions (B) of the $\mathrm{H}_{2} \mathrm{O}$ and PCA extracts of RA/SM reacted with anti-CEA to give double precipitin lines. One of these appeared to form a line of partial identity with tumour CEA in the adjacent well, indicating a common antigenic determinant. These reactions with anti-CEA and the RA/SM fractions were obtained despite very low CEA concentrations as measured by the radioimmunoassay. Since tumour CEA did not produce visible immunoprecipitins at these concentrations with the same conditions, it might imply that the CEA activity in the high molecular weight RA/SM fractions was underestimated in the radioimmunoassay. This could happen if the antigen were bound in an aggregate rendering some of its sites unavailable for reaction with antibody in the radioimmunoassay. Alternatively, CEA antigenic determinants in RA/SM extracts may form part of the surface determinants of a larger molecule. Either of these possibilities could facilitate immunoprecipitation of low amounts of the antigen.

Although CEA activity in RA/SM appears to have quite different properties from that in tumour and $\mathrm{N}$ lung in terms of solubility in perchloric acid and molecular weight, much of this might be explained by an association with large molecular weight material. Such material may be underestimated in the radioimmunoassay and be precipitated during 
acid treatment. The use of strong dissociating media may help to resolve this.

With an antiserum to the CEA-like components in RA/SM, further identification of their relationship to tumour CEA may be made and more sensitive methods for their detection and measurement could be developed, thus allowing a better judgement of whether or not they represent the expression of neoantigens in the rheumatoid synovium.

The goat anti-CEA (4G3), anti-CEX (8G3), and CEA were kindly donated by Drs. D. A. Darcy and C. Turberville, Chester Beatty Research Institute, London. Financial support from the Arthritis and Rheumatism Council is gratefully acknowledged.

\section{References}

Darcy, D. A., Turberville, C., and James, R. (1973). Immunological study of carcinoembryonic antigen (CEA) and a related glycoprotein. British Journal of Cancer, 28, $147-160$.

Dyce, B. J., and Haverback, B. J. (1974). Free and bound carcinoembryonic antigen in neoplasms and in normalO adult and fetal tissue. Immunochemistry, 11, 423-430.

Egan, M. L., Lautenschleger, J. T., Coligan, J. E., and Todd, $\Rightarrow$ C. W. (1972). Radioimmune assay of carcinoembryonic antigen. Immunochemistry, 9, 289-299.

Feizi, T., Turberville, C., and Westwood, J. H. (1975) Blood-group precursors and cancer-related antigens. Lancet, 2, 391-393.

Kleist, S. von, Chavanel, G., and Burtin, P. (1972). Identi- $\mathbb{D}$ fication of a normal antigen that crossreacts with the carcinoembryonic antigen (CEA). Proceedings of the National Academy of Sciences of the U.S.A., 69, 2492-2494. $\vec{\circ}$

Laurence, D. J. R., Stevens, U., Bettelheim, R., Darcy, D., Leese, C., Turberville, C., Alexander, P., Johns, E. W., $\vec{\omega}$ and Munro Neville, A. (1972). Role of plasma carcino-embryonic antigen in diagnosis of gastrointestinal, mam-0 mary and bronchial carcinoma. British Medical Journal, $3,605-609$.

Simmons, D. A. R., and Perlmann, P. (1973). Carcino-. embryonic antigen and blood group substances. Cancer Research, 33, 313-322.

Unger, A., Panayi, G. S., and Lessof, M. H. (1974). Carcino-O embryonic antigen in rheumatoid arthritis. Lancet 1, 781-783.

Unger, A., Panayi, G. S., and Lessof, M. H., (1975). Carcinoembryonic antigen in rheumatic diseases. Rheumatism 음 and Rehabilitation, 14, 19-24. 